\title{
Modelización del transporte marítimo de contenedores
}

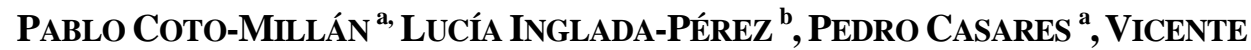 \\ INGLADA LÓPEZ DE SABANDO ${ }^{c}$ \\ a Universidad de Cantabria, Fctad. CC.EE. y EE. Avda. de los Castros, s/n, 39005 Santander, \\ España.E-mail: cotop@unican.es, casaresp@unican.es \\ ${ }^{b}$ Universidad Carlos III de Madrid, Fctad. CC.SS y J., c/ Madrid, 126, 28903 Getafe (Madrid), \\ España.E-mail: linglada@eco.uc3m.es \\ c Universidad Nacional de Educación a Distancia (UNED),Fctad. CC.EE. y EE., Paseo Senda del \\ Rey, 11, 28040 Madrid, España.E-mail: vinglada@cee.uned.es
}

\begin{abstract}
RESUMEN
El objetivo de esta investigación es analizar el comportamiento del tráfico mundial de contenedores durante las últimas décadas estudiando su comportamiento respecto al ciclo económico. Para ello se estima un modelo econométrico dinámico para un panel de 49 países durante el periodo 2000-2016 que nos permite identificar las relaciones en el corto y largo plazo entre el tráfico marítimo de contenedores y la actividad económica medida por el volumen del comercio internacional total y del comercio internacional de mercancías.
\end{abstract}

Palabras clave: Transporte de contenedores, Modelos de panel, Comercio internacional.

\section{Modeling of Containerized Maritime Transport}

\begin{abstract}
The objective of this research is to analyze the behavior of global container traffic during the last decades by studying its behavior with respect to the economic cycle. For this, a dynamic econometric model is estimated for a panel of 49 countries during the period 2000-2016 that allows us to identify the short and long term relationships between maritime container traffic and economic activity measured by the volume of total international trade and international merchandise trade.
\end{abstract}

Keywords: Panel Models, Container Transportation, International Commerce.

Clasificación JEL: E10

Artículo recibido en julio de 2018 y aceptado en septiembre de 2018

Artículo disponible en versión electrónica en la página www.revista-eea.net, ref. ə-36309 


\section{INTRODUCCIÓN}

La innovación tecnológica asociada a la utilización del contenedor como unidad estandarizada de carga ha supuesto una revolución en el transporte de mercancías debido a sus múltiples ventajas en forma de reducción de coste y tiempo en el transporte y en particular, en los procesos de carga y descarga. Desde su tímida irrupción en 1956, su difusión ha sido vertiginosa comparada con otras innovaciones en el transporte (Rua, 2014). Como consecuencia de la tendencia creciente en la utilización del contenedor en los flujos internacionales de mercancías, el transporte marítimo de contenedores ha crecido en las últimas décadas de forma exponencial, triplicando su volumen de tráfico entre 2000 y 2016 con un ritmo de crecimiento superior al correspondiente a la renta y el comercio mundial. Bernhofen y otros (2016) sostienen que el rápido desarrollo del transporte de contenedores ha sido uno de los principales factores que han contribuido al fenómeno de la globalización en la economía mundial.

Dado el interés que presenta para todo el sistema portuario, no es de extrañar los numerosos trabajos que han estudiado con detalle y realizado predicciones sobre el tráfico de contenedores en determinados puertos. (Ver, por ejemplo, Fung, 2002; Hui y otros, 2004; y Peng y Chu, 2009). Sin embargo, a pesar de la importante contribución del transporte marítimo en contenedores al desarrollo del comercio y de la economía mundial, existe un déficit de investigaciones sobre las relaciones entre actividad comercial y tráfico de contenedores a nivel global y desde una perspectiva macroeconómica (Luo y otros, 2009).

El objetivo de esta investigación es dar una respuesta basada en la evidencia empírica al interrogante de si existe una relación en el largo plazo entre el transporte marítimo de contenedores $(\mathrm{CON})$ y el comercio internacional total (COM) y de mercancías (MER). La herramienta metodológica empleada es el análisis de cointegración con datos de panel que nos permite obtener elasticidades a corto y largo plazo entre las variables consideradas. Para ello se propone un modelo que explica el efecto del comercio internacional sobre el volumen del transporte marítimo de contenedores El modelo propuesto se contrasta estadísticamente para un panel de 49 países durante el periodo 2000-2016. Los resultados obtenidos sobre la magnitud de la elasticidad respecto a las variables COM y MER son positivos y significativos estadísticamente Además respecto a la variable MER la magnitud de la elasticidad es sensiblemente mayor que la unidad Este modelo nos permite predecir escenarios futuros sobre la evolución del transporte marítimo de contenedores en función de comportamiento del comercio de mercancías.

Con estos objetivos, la estructura de esta investigación es la siguiente. La sección segunda se destina a la descripción de los datos empleados y al análisis de la evolución global de las series CON, COM y MER durante el periodo de referencia., destacando los principales rasgos de su evolución, así como las 
diferencias entre grupos de países y periodos de tiempo, en especial durante la reciente crisis económica mundial. En la sección tercera se describen los principales rasgos de la metodología utilizada en la investigación. A continuación, en la sección cuarta se discuten los resultados obtenidos, y por último, en la sección quinta se exponen las principales conclusiones.

\section{DATOS}

La selección de las variables utilizadas está basada en la literatura existente y en la disponibilidad de datos. La variable dependiente en ambos modelos es el transporte marítimo de contenedores medido a través de las TEU transportadas (TEU: unidades equivalentes a 20 pies) Se consideran como variables explicativas al comercio internacional total y al comercio internacional de mercancías, respectivamente en cada modelo. Los datos usados en esta investigación, referentes al comercio total y de mercancías, así como al tráfico de contenedores para los 49 países incluidos en la muestra cubren el periodo 2000-2016 y se han obtenido del Banco Mundial (World Development Indicators). La selección de los países de la muestra se ha basado en la disponibilidad de datos, En la Tabla 1 se muestran los principales estadísticos de las variables utilizadas.

Tabla 1

Estadísticos descriptivos de las variables utilizadas

\begin{tabular}{|c|c|c|c|c|c|}
\hline Variables & Observaciones & Media & $\begin{array}{c}\text { Desviación } \\
\text { típica }\end{array}$ & Mínimo & Máximo \\
\hline $\begin{array}{c}\text { CON } \\
\text { (Miles de TEUs) }\end{array}$ & 833 & 8566,3 & 18900,9 & 14,1 & 199565,5 \\
\hline $\begin{array}{c}\text { COM } \\
\text { (Miles de millones de US\$ }\end{array}$ & 829 & 58252,1 & 79769,6 & 1036,2 & 487913,4 \\
\hline $\begin{array}{c}\text { MER } \\
\text { (Miles de Millones de US\$) }\end{array}$ & 832 & 48020,9 & 66555,5 & 499,5 & 374347,3 \\
\hline
\end{tabular}

Fuente: Elaboración propia.

Para reducir posibles problemas de heterocedasticidad en las estimaciones y estimar elasticidades con mejor interpretación económica, todas las series se transforman en logaritmos.

En la Figura 1 se muestra la evolución de tráfico marítimo de contenedores en diferentes países o grupos de países seleccionados por su relevancia en el comercio internacional durante el periodo 2000-2016. Se observa que existe un crecimiento tendencial, excepto durante 2008 donde desciende bruscamente como consecuencia de la profunda crisis económica mundial. El ritmo de crecimiento de la serie analizada es superior en el caso de China respecto a la Unión Europea y Estados Unidos. 
Figura 1

Evolución del tráfico marítimo de contenedores en diferentes países (Valor 100 en 2000)

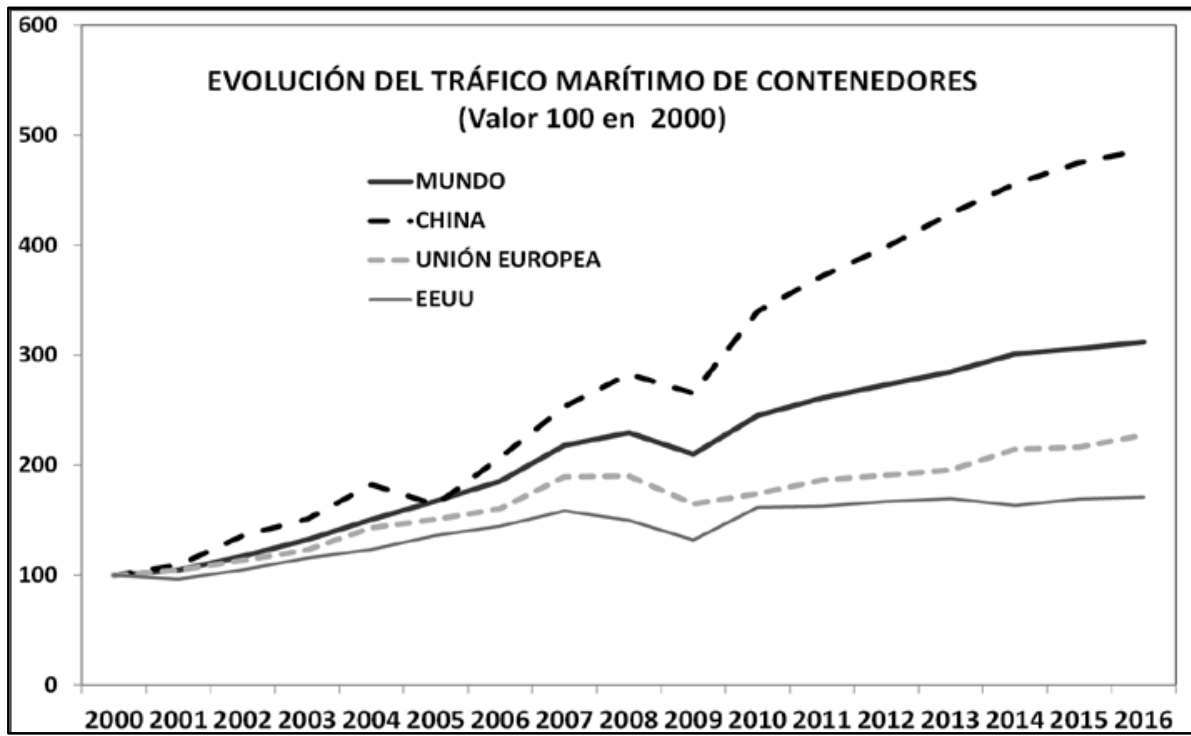

Fuente: Elaboración propia.

Tabla 2

Variación del tráfico marítimo de contenedores en diferentes periodos y grupos de países (Tasa media anual acumulativa en tanto por ciento)

\begin{tabular}{|l|c|c|c|c|}
\hline & $\mathbf{2 0 0 0 - 0 8}$ & $\begin{array}{c}\mathbf{2 0 0 8 - 0 9} \\
\text { (Crisis) }\end{array}$ & $\mathbf{2 0 0 9 - 1 6}$ & $\mathbf{2 0 0 0 - 1 6}$ \\
\hline Mundial & $10,95 \%$ & $-8,52 \%$ & $5,82 \%$ & $7,37 \%$ \\
\hline Unión Europea & $8,35 \%$ & $-13,33 \%$ & $4,72 \%$ & $5,27 \%$ \\
\hline China & $13,88 \%$ & $-6,16 \%$ & $9,05 \%$ & $10,40 \%$ \\
\hline Estados Unidos & $5,19 \%$ & $-11,93 \%$ & $3,76 \%$ & $3,41 \%$ \\
\hline OCDE & $7,37 \%$ & $-11.68 \%$ & $4,90 \%$ & $4,99 \%$ \\
\hline Países con renta baja & $14,37 \%$ & $0,46 \%$ & $6,92 \%$ & $10,15 \%$ \\
\hline Países con renta alta & $8,78 \%$ & $-11,21 \%$ & $3,93 \%$ & $5,29 \%$ \\
\hline
\end{tabular}

Fuente: Elaboración propia.

En la Tabla 2 se muestra con mayor detalle la variación del tráfico marítimo de contenedores en diferentes periodos de tiempo y para varios grupos de países. Se observa que el impacto de la reciente crisis mundial sobre el tráfico mundial es un descenso del 8,52\%. Este impacto es especialmente pronunciado en los países de renta alta y concretamente en los casos de Estados Unidos (-11,93\%), y la Unión Europea (-13,33\%). Por el contrario, su magnitud es 
menor en China (-6,16\%) y sobre todo en los países de renta baja donde incluso el tráfico no desciende en dicho periodo (0,46\%). Durante todo el periodo de estudio 2000-2016 el tráfico mundial de contenedores ha crecido con una tasa anual media acumulativa de 7,37\%, multiplicándose por 3 en dicho periodo. Asimismo, el ritmo de crecimiento es mayor en el periodo pre crisis 2000-2008 (10,95\%) que en el periodo post crisis (5,82\%).

Figura 2

Evolución en 2000-2016 del tráfico marítimo de contenedores y del comercio mundial (valor 100 en 2000)

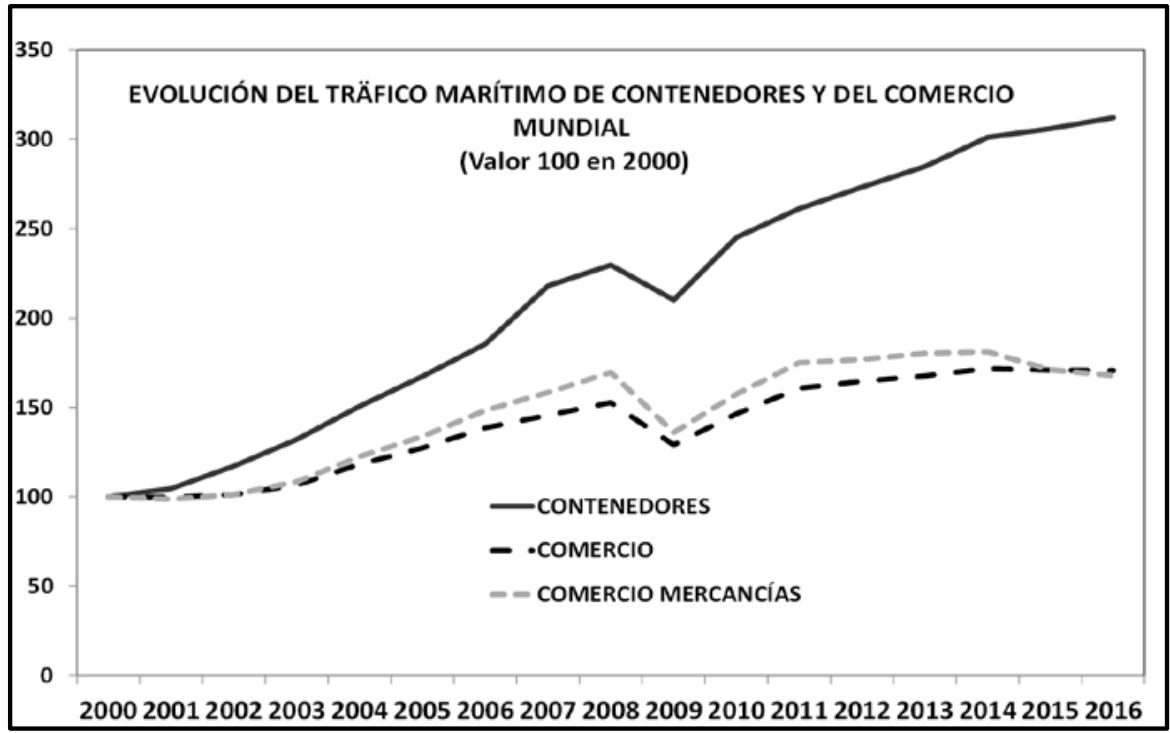

Fuente: Elaboración propia.

En la Figura 2 se representa la evolución del tráfico marítimo mundial de contenedores junto al comercio total y de mercancías, durante 2000-2016. Se observan unas pautas de evolución muy similares en las tres series con un pronunciado descenso en el año 2008. En los últimos años se observa una leve contracción del comercio mundial que de acuerdo con UNCTAD (2016), se debe a diferentes causas como la lenta recuperación de las economías europeas, la debilidad de las inversiones mundiales, y la desaceleración de las grandes economías en desarrollo.

\section{METODOLOGÍA}

La expresión genérica de un modelo para datos de panel como el que se utiliza en esta investigación es la siguiente: 


$$
y_{i t}=\alpha_{i t}+\beta_{1} x_{1 i t}+\beta_{2} x_{2 i t}+\ldots \ldots \beta_{k} x_{k i t}+\varepsilon_{i t}
$$

tal que:

$i=1,2 \ldots . . \mathrm{N}$ significa la unidad transversal (país) $i$-ésima

$t=1,2 \ldots \ldots \ldots . . .$. T es el tiempo (años).

El proceso de análisis de cointegración para datos de panel desarrollado en esta investigación comienza comprobando cuál es el orden de integración de las variables a través de la aplicación de contrastes de raíz unitaria para datos de panel. Posteriormente se estudia la posibilidad de que exista una relación estable y de largo plazo entre las mismas. En otras palabras, se investiga la posibilidad de que exista cointegración entre tales variables. Finalmente se estiman las relaciones de largo plazo o modelo de corrección de error.

\subsection{Existencia de raíz unitaria}

En primer lugar es necesario contrastar la existencia de raíces unitarias para comprobar que las variables utilizadas no son estacionarias pero que después de aplicar el operador diferencia todas las nuevas variables ya son estacionarias. La utilización de este tipo de contrastes se justifica por Banerjee (1999); Baltagi y Kao (2000) y Banerjee et al. (2005),entre otros, que sugieren que los contrastes de raíces unitarias basados en datos de panel tienen mayor potencia que los contrastes basados en series individuales.

Existen varios contrastes de raíces unitarias para datos de panel que parten de diferentes supuestos ${ }^{1}$. En esta investigación utilizamos dos test diferentes que permiten la utilización de paneles incompletos como es nuestro caso. Los test utilizados son: Im, Pesaran y Shin (2003) (IPS) y de tipo Fischer (Choi 2001). Ambos plantean como hipótesis nula la presencia de una raíz unitaria en las series analizadas. El test IPS permite que dicho parámetro varíe de forma libre entre las distintas unidades, países en nuestro caso, por lo que la hipótesis alternativa planteada en estos casos es la existencia de alguna serie estacionaria en el conjunto total.

\subsection{Existencia de cointegración}

Para evitar el problema de la regresión espuria y comprobar si las variables de nuestro modelo están cointegradas y, por lo tanto, evolucionan conjuntamente en el largo plazo, es necesario utilizar un contraste específico de cointegración para datos de panel que tenga en cuenta no solo la dimensión temporal sino también la transversal. Para ello en esta investigación se utilizan los cuatro test definidos por Westerlund (2007) que son una extensión de los de Banerjee et al. (1998) y

\footnotetext{
${ }^{1}$ En Breitung y Pesaran (2007) se lleva a cabo una revisión extensa de literatura sobre raíces unitarias y cointegración en paneles.
} 
que poseen la gran virtud de ser robustos ante la presencia de una relación de dependencia transversal entre los diferentes países, como de hecho ocurre en nuestro modelo y permiten relajar la restricción de factor común al no basarse en el examen de los residuos.

Concretamente, para verificar la hipótesis nula de inexistencia de cointegración en un panel de datos, Westerlund (2007) propone cuatro test consistentes, básicamente, en verificar si el término de corrección del error de un modelo de corrección del error condicional es estadísticamente nulo. De forma que, si la hipótesis nula de no corrección del error es rechazada, también habría de rechazarse la hipótesis de no cointegración (Baltagi, 2008). De los cuatro test de Westerlund), dos de ellos, $P_{\tau}$ y $P_{\sigma}$, son estadísticos de panel que tienen como hipótesis alternativa, frente a la nula de no cointegración, que el panel en su conjunto está cointegrado, mientras que $G_{\tau}$ y $G_{\alpha}$ son estadísticos de media de grupo que permiten contrastar si al menos alguna de unidades del panel presenta cointegración. Los cuatro contrastes permiten la inclusión de constante y tendencia en la relación de cointegración.

Las expresiones de los cuatro estadísticos son las siguientes ${ }^{2}$ :

$$
\begin{aligned}
G_{\tau} & =\frac{1}{N} \sum_{i=1}^{N} \frac{\hat{\alpha}_{i}}{S E\left(\hat{\alpha}_{i}\right)} & G_{\alpha} & =\frac{1}{N} \sum_{i=1}^{N} \frac{T\left(\hat{\alpha}_{i}\right)}{\hat{\alpha}_{i}(1)} \\
P_{\tau} & =\frac{\hat{\alpha}}{S E(\hat{\alpha})} & P_{\alpha} & =T \hat{\alpha}
\end{aligned}
$$

Donde $\hat{\alpha}_{i}$ representa el coeficiente de corrección del error, $\hat{\alpha}_{i}(1)=1-\sum_{j=1}^{\rho_{i}} \hat{\alpha}_{i j}$ y $S E$ simboliza la desviación típica.

En todos los test la hipótesis nula de inexistencia de cointegración se contrasta verificando que el término de corrección de error en el correspondiente modelo de corrección del error condicional es estadísticamente gual a cero (Baltagi, 2008).

Se supone que la expresión del modelo de corrección de error es la siguiente:

$$
\Delta y_{i t}=\delta_{i} d_{t}+\alpha_{i}\left(y_{i, t-1}-\beta_{i} x_{i, t-1}\right)+\sum_{j=1}^{p_{i}} a_{i j} \Delta y_{i, t-j}+\sum_{j=0}^{p_{i}} b_{i j} \Delta x_{i, t-j}+u_{i, t}
$$

Donde el parámetro $\alpha_{i}$ mide la velocidad del ajuste hasta el equilibrio en el largo plazo. Se requiere que el dicho parámetro sea distinto de cero y negativo para que exista una relación de cointegración entre las variables del modelo.

\footnotetext{
${ }^{2}$ La información detallada sobre la definición de los términos que integran estos estadísticos puede encontrarse en Westerlund (2007).
} 


\subsection{Estimación de relaciones del largo plazo}

Una vez comprobada la existencia de una relación de cointegración entre las variables que integran nuestro modelo, la etapa final consiste en estimar el modelo de corrección de error que nos permitirá obtener las elasticidades de largo plazo del output respecto a las variables explicativas. Asimismo, con la estimación de dicho modelo podernos estimar la velocidad de ajuste hacia la posición de equilibrio en el largo plazo.

El modelo que se va estimar en el largo plazo se basa en que la demanda para el transporte marítimo de contenedores es una demanda derivada del comercio internacional que, a su vez, es determinado por las ventajas comparativas entre los diferentes países. (Luo y otros, 2009). En resumen, suponemos que la función a estimar en el largo plazo se adaptaría a la expresión:

$$
\mathrm{CON}_{t}=A_{t} \cdot \mathrm{COMER} R_{t}^{\alpha}
$$

O bien tomando logaritmos:

$$
\operatorname{con}_{i t}=a_{i}+\alpha_{i} \text { comer }_{i, t}+u_{i, t}
$$

Donde:

$\mathrm{con}_{i t}$ es el logaritmo del volumen de tráfico marítimo de contenedores;

comer $_{i t}$ es el logaritmo de la variable explicativa en términos reales. Se consideran dos variables alternativas: Comercio exterior total (COM) y comercio de mercancías (MER);

$i=1 \ldots \ldots . . . . . . N$ es el número de países;

$t=1$.............T es el número del año correspondiente.

La estimación eficiente de la relación de cointegración en el largo plazo en un panel de datos es factible en el caso de variables no estacionarias cointegradas En este sentido, se han propuesto varios tipos de estimadores para un panel de datos que son en su mayoría extensiones o ampliaciones de los métodos tradicionales de series temporales. Para estimar nuestro modelo de panel de datos cabe utilizar tres tipos de estimadores. El primero es el estimador dinámico de efectos fijos (DFE). Este estimador solo permite a la constante diferir entre las unidades del panel mientras que los otros parámetros y la varianza del término residual se restringen a que sean idénticas para todas las unidades. Pero cabe resaltar que si los coeficientes no fueran homogéneos entre los diversos grupos, el estimador DFE es insesgado incluso para elevados $\mathrm{T}$.

Otro estimador alternativo -Mean Group (MG)- consiste básicamente en realizar la regresión para cada grupo y obtener la media aritmética de los coeficientes obtenidos en el paso anterior. Este estimador no sólo permite a la constante variar entre las unidades sino también a los coeficientes y las varianzas. 
Finalmente Pesaran y otros (1999) proponen un estimador de máxima verosimilitud que combina los dos anteriores $M G$ y $D F E$, el denominado pooled mean group ( $P M G)$ para paneles dinámicos heterogéneos que es una extensión a un panel de datos del modelo $\mathrm{ARDL}^{3}$. El procedimiento utilizado consiste básicamente en estimar un modelo ARDL a partir de los datos, que puede ser transformado en una ecuación de corrección de error para facilitar su interpretación económica. Este estimador PMG obliga a que los coeficientes de la relación de largo plazo sean idénticos para cada unidad ${ }^{4}$ pero permite que la constante, los coeficientes en el largo plazo, en nuestro caso elasticidades, y las varianzas de los términos residuales varíen entre los países (Pesaran y otros, 1999). Con este estimador podemos estimar eficientemente las relaciones en el largo plazo mientras suministra información sobre el comportamiento en el corto plazo (por ejemplo los impactos contemporáneos y la velocidad de ajuste al equilibrio). Finalmente, otra ventaja es que mientras se supone que los coeficientes de largo plazo son homogéneos (idénticos en cada unidad del panel) se permite que los coeficientes del corto plazo sean específicos de cada país.

La elección del estimador entre los tres citados se lleva a cabo mediante el test de Hausman. En primer lugar se comparan los estimadores PMG y $M G$. Para ello hay que tener en cuenta que si las elasticidades a largo plazo son iguales para todos los países, el estimador $P M G$ es consistente y eficiente por lo que dicho estimador es el elegido. Por el contario, si se rechaza la hipótesis de igualdad entre coeficientes, se debe utilizar el estimador $M G$ que es consistente en cualquier caso. El test de elección entre ambos modelos se lleva a cabo con el test de Hausman. A continuación se elige entre los estimadores $M G$ o $P M G$ y $D F E$. Para ello hay que tener en cuenta que el estimador DFE está sujeto a un sesgo generado por la posible existencia de endogeneidad entre el término de error y la variable dependiente retardada. En este caso el test de Hausman se utiliza para medir su tamaño. Si no fuera significativo se elegiría el estimador DFE.

\section{ANÁLISIS DE RESULTADOS}

El marco metodológico definido anteriormente se aplica para estimar las elasticidades precio en el corto y largo plazo del transporte marítimo de contenedores respecto al comercio internacional total y al de mercancías,

\footnotetext{
${ }^{3}$ Autoregressive distributed lag.

${ }^{4}$ Según Breitung y Pesaran (2007), a menudo la teoría económica predice la existencia de la misma relación de cointegración para un conjunto de unidades transversales, por lo que esta restricción sobre los coeficientes a largo plazo no se alejaría excesivamente de lo que sucede en la realidad. Esta metodología es apropiada para paneles no estacionarios donde $N$ y $T$ son relativamente grandes. Por ejemplo, Pesaran y al. (1999) aplican esta metodología a dos ejemplos con las dimensiones siguientes: (i) $T=32$ y $N=24$; y (ii) $T=17$ y $N=10$.
} 


\subsection{Existencia de raíces unitarias}

En la Tabla 3 se muestran los resultados obtenidos para los dos tipos de contrastes de raíz unitaria (IPS y Fischer) que son adecuados para datos de panel incompleto como es nuestro caso. Se observa en las columnas de dicha tabla donde aparecen los p-valores que para las tres variables analizadas no podemos rechazar la hipótesis nula de existencia de raíz unitaria a favor de la hipótesis de que sean estacionarias a un nivel de significación del 5\%. Sin embargo para las variables obtenidas después de aplicar el operador diferencia podemos rechazar la hipótesis nula de existencia de raíz unitaria a favor de la hipótesis de que sean estacionarias a un nivel de significación del 5\%. Cabe concluir, por tanto que las tres variables son $\mathrm{I}(1)$.

Tabla 3

Contrastes de panel de raíces unitarias y orden de integración

\begin{tabular}{|c|c|c|c|}
\hline Variable & $\begin{array}{c}\text { IPS } \\
\text { (p-valor) }\end{array}$ & $\begin{array}{c}\text { Fischer } \\
\text { (p-valor) }\end{array}$ & $\begin{array}{c}\text { Orden de } \\
\text { integración }\end{array}$ \\
\hline con & 0,155 & 0,564 & $\mathrm{I}(1)$ \\
\hline com & 0,216 & 0,773 & $\mathrm{I}(1)$ \\
\hline mer & 0,323 & 0,285 & $\mathrm{I}(1)$ \\
\hline$\Delta$ con & 0,000 & 0,000 & - \\
\hline$\Delta$ com & 0,000 & 0,003 & - \\
\hline$\Delta$ mer & 0,000 & 0,000 & - \\
\hline
\end{tabular}

Nota: $\Delta$ es el operador primera diferencia. Todas las variables están en forma logarítmica.

Fuente: Elaboración propia.

\subsection{Contraste de existencia de cointegración}

Los resultados obtenidos para los 4 estadísticos de Westerlund sobre la existencia de cointegración se presentan en las Tablas 4 y 5 para los dos modelos M1 y M2 correspondientes a las variables explicativas com y mer, respectivamente. Debido a que los resultados pueden verse afectados por la amplitud del kernel elegido se ha empleado la fórmula de Wesrerlund con el fin de determinar el ancho de banda para el estimador Kernel de Barlett de las varianzas en el largo plazo.

Se observa en la última columna de dichas tablas, donde aparecen los pvalores, que la hipótesis nula de inexistencia de cointegración se rechaza con un nivel de significación del $5 \%$ en todos los casos excepto en uno para el estadístico $\mathrm{G}_{\mathrm{t}}$ en la Tabla 5. Por lo tanto, cabe concluir que las variables del panel están cointegradas y por tanto podemos estimar el modelo de corrección del error y la relación de equilibrio en el largo plazo en ambos casos. 
Tabla 4

Resultados del Test de Westerlund para cointegración (Modelo M1 con variable explicativa com).

\begin{tabular}{|c|c|c|c|}
\hline Estadístico & Valor & z.valor & p-valor \\
\hline $\mathrm{G}_{\mathrm{T}}$ & $-2,160$ & $--2,983$ & $0,001^{\star \star \star}$ \\
\hline $\mathrm{G}_{\alpha}$ & $-8,252$ & $-1,427$ & $0,077^{*}$ \\
\hline $\mathrm{P}_{\mathrm{T}}$ & $-12,707$ & $-2,589$ & 0,005 *** \\
\hline $\mathrm{P}_{\alpha}$ & 6,967 & $-4,315$ & 0,000 *** \\
\hline
\end{tabular}

Notas: Los símbolos ***, ** $y$ * indican, respectivamente, que el parámetro es significativo estadísticamente con un nivel de significación 1\%, 5\% y $10 \%$.

Fuente: Elaboración propia.

Tabla 5

Resultados del Test de Westerlund para cointegración (Modelo M2 con variable explicativa mer).

\begin{tabular}{|c|c|c|c|}
\hline Estadístico & Valor & z.valor & p-valor \\
\hline $\mathrm{G}_{\mathrm{T}}$ & $-1,900$ & $-0,955$ & 0,170 \\
\hline $\mathrm{G}_{\alpha}$ & $-8,781$ & $-2,107$ & $0,018^{\star *}$ \\
\hline $\mathrm{P}_{\mathrm{T}}$ & $--12,792$ & $-2,674$ & $0,004^{\star * *}$ \\
\hline $\mathrm{P}_{\alpha}$ & $-7,949$ & $-5,863$ & 0,000 *** \\
\hline
\end{tabular}

Notas: Los símbolos ***, ** $y$ * indican, respectivamente, que el parámetro es significativo estadísticamente con un nivel de significación 1\%, 5\% y $10 \%$.

Fuente: Elaboración propia.

\subsection{Estimación del modelo}

Como se ha mencionado anteriormente, en los dos modelos se han considerado tres posibles estimadores: pooled mean group ( $P M G)$ que impone efectos comunes en el largo plazo, mean group $(M G)$ que permite a los coeficientes del largo plazo ser diferentes para cada unidad del panel, y Dynamic Fixed Effects (DFE) que tiene la restricción de que todos los coeficientes de las variables explicativas y las varianza del error han de ser iguales para todas las unidades. La elección entre dichos estimadores se realiza a través del test de Hausman. De acuerdo con dicho test se elige el estimador $\mathrm{PMG}^{5}$.

En la Tabla 3 se muestran los resultados obtenidos para el modelo de corrección de error para los dos modelos y con el estimador seleccionado. Entre dichos resultados cabe destacar que para los dos modelos el coeficiente de ajuste es negativo y significativo estadísticamente al nivel de significación del

\footnotetext{
${ }^{5}$ Modelo M1: Entre PMG y MG se ha obtenido para el estadístico de Hausman chi2(1) = 0,32 y Prob $>$ chi2 = 0,574; elegimos PMG. Entre PMG y DFE se ha obtenido para el estadístico de Hausman chi2(1) $=3,86$ y Prob $>$ chi2 $=0,049$. Luego finalmente se elige el estimador PMG.

Modelo M2: Entre PMG y MG se ha obtenido para el estadístico de Hausman chi2(1) = 1,16 y Prob $>$ chi2 $=0$,282: elegimos PMG. Entre PMG y DFE se ha obtenido para el estadístico de Hausman chi2(1) $=6,35$ y Prob>chi2 = 0,012. Luego finalmente se elige el estimador PMG.
} 
$1 \%$, lo que está conforme con la existencia de una relación de largo plazo en ambos modelos. Este coeficiente, que mide la velocidad del ajuste hacia el nivel de equilibrio después de un shock que desplace al modelo de su equilibrio, varía entre 0,241 en el modelo M1 que tiene como variable explicativa al comercio internacional total y 0,186 para el modelo M2 que tiene como variable explicativa al comercio internacional de mercancías. Es decir, la magnitud del ajuste inicial es del $24,1 \%$ en el caso del modelo M1, que incluye como variable explicativa al volumen total de comercio, y del 18,6\% para modelo M2 que incluye como variable explicativa al volumen del comercio de mercancías.

Si nos fijamos en la elasticidad a largo plazo del tráfico marítimo de contenedores respecto al comercio internacional, que es la que presenta mayor interés, cabe destacar que su magnitud varía desde 0,929 en el modelo M1, que tiene como variable explicativa al comercio internacional total, a 1,879 para el modelo M2 que tiene como variable explicativa al comercio internacional de mercancías. En ambos casos es significativa estadísticamente al 1\% y presenta el signo esperado. Cabe deducir, por tanto, que existe un impacto positivo del comercio sobre el tráfico de contenedores. Un incremento del volumen de comercio de mercancías del $10 \%$ produciría en el largo plazo un aumento del $18,79 \%$ en el tráfico marítimo de contenedores. Asimismo, las correspondientes elasticidades en el corto plazo respecto al comercio total y al comercio de mercancías son positivas en ambos modelos y significativamente diferente de cero a un nivel del 5\% y del 10\% para el modelo M1 y M2, respectivamente.

Tabla 6

Resultados de la estimación del modelo de corrección de error

\begin{tabular}{|c|c|c|c|c|c|c|}
\hline \multirow[b]{2}{*}{ Variables } & \multicolumn{3}{|c|}{ Modelo M1 (Con COM) } & \multicolumn{3}{|c|}{ Modelo M2 (Con MER) } \\
\hline & Coeficiente & $\mathbf{z}$ & $P>|z|$ & Coeficiente & $\mathbf{z}$ & $P>|z|$ \\
\hline \multicolumn{7}{|l|}{ Corto plazo } \\
\hline Corrección de error & $-0,241^{\star * *}$ & $-8,61$ & 0,000 & $-0,186^{\star \star \star}$ & $-4,68$ & 0,000 \\
\hline C & $-3,223^{\star * *}$ & $-8,42$ & 0,000 & $-7,856^{\star \star \star}$ & $-4,63$ & 0,000 \\
\hline$\Delta \mathrm{COM}$ & $0,243^{\star *}$ & 2,74 & 0,006 & -------- & ------ & ------ \\
\hline$\Delta \mathrm{MER}$ & ---------- & ---- & ------- & $0,123^{*}$ & 1,69 & 0,091 \\
\hline \multicolumn{7}{|l|}{ Largo Plazo } \\
\hline COM & $0,929 * \star *$ & 29,50 & 0,000 & ------ & ----- & ----- \\
\hline MER & -------- & ------ & ----- & $1,879 * \star \star$ & 26,77 & 0,000 \\
\hline
\end{tabular}

Notas: Los símbolos ***, ** $y$ * indican, respectivamente, que el parámetro es significativo estadísticamente con un nivel de significación $1 \%, 5 \%$ y $10 \%$.

Todas las variables consideradas están expresadas en logaritmos.

Fuente: Elaboración propia. 


\section{CONCLUSIONES}

En este trabajo se estudia la existencia de una relación en el largo plazo entre el transporte marítimo de contenedores y el comercio internacional, medido alternativamente por el volumen de comercio total y por el volumen de comercio de mercancías. El modelo propuesto se contrasta estadísticamente para un panel de 49 países con datos anuales durante el periodo 2000-2016. La herramienta del análisis de cointegración para datos de panel nos permite además obtener las elasticidades en el corto y largo plazo, una vez comprobada la existencia de una relación de cointegración entre las dos variables.

Los resultados de la estimación son satisfactorios presentando todas las elasticidades, así como el término de corrección del error o ajuste los signos esperados y además todos ellos son significativos estadísticamente. Estos resultados están en la línea de los resultados obtenidos por Coto-Millán et al. (2018 y 2013).

Las magnitudes de las elasticidades en el largo plazo del transporte marítimo de contenedores respecto al volumen de comercio total mundial y comercio de mercancías son 0,939 y 1,879, respectivamente. Este último valor puede interpretarse como que un $10 \%$ de crecimiento en el comercio de mercancías se traduciría en un aumento del volumen del tráfico de contenedores en el 18,8\%. Por tanto, al ser la magnitud de la elasticidad nítidamente superior a la unidad, cabe concluir que existe un impacto positivo del comercio sobre el tráfico de contenedores y que el ritmo de crecimiento del tráfico de contenedores superaría al del comercio de mercancías.

Los resultados obtenidos y las consiguientes conclusiones deducidas en esta investigación pueden servir de guía eficaz para el conocimiento de cuál será la evolución en el largo plazo del tráfico de contenedores ante los diversos escenarios existentes sobre la evolución futura del comercio internacional. Estos resultados presentan interés para todos los agentes que forman parte del mercado de transporte marítimo, incluyendo, entre otros, a armadores, consignatarios o intermediarios comerciales y financieros. Asimismo, pueden contribuir al diseño de la política de inversiones y a la gestión eficiente en el ámbito portuario.

Las investigaciones futuras podrían estar encaminadas a estimar el modelo propuesto para determinados grupos de países, seleccionados de acuerdo con criterios de renta o por bloques comerciales, como es el caso de la Unión Europea. También hacia la consideración en el modelo de otras variables explicativas como la estructura sectorial, el PIB o el desglose del comercio entre importaciones y exportaciones suprimiendo determinado productos no susceptibles de transporte en contenedores como es el caso de los hidrocarburos. 


\section{REFERENCIAS BIBLIOGRÁFICAS}

BALTAGI, B.H.; KAO, C. (2000). "Nonstationary panels, cointegration in panels and dynamic panels: a survey". Advances in Econometrics, 15, pp. 7-51.

BALTAGI, B. (2008). Econometric analysis of panel data. New Jersey: John Wiley \& Sons, Chichester.

BANERJEE, A.; DOLADO, J.J.: MESTRE, R. (1998). "Error-Correction Mechanism Tests for Cointegration in a Single-Equation Framework". Journal of Time Series Analysis, 19(3), pp. 267-283.

BANERJEE, A. (1999). "Panel unit roots and cointegration: An overview". Oxford Bulletin of Economics and Statistics, 61, pp. 607-29.

BANERJEE, A.; MARCELLINO, M.; OSBAT, C. (2005). "Testing for PPP: Should we use panel methods?". Empirical Economics, 30(1), pp. 77-91.

BERNHOFEN, D.M.; EL-SAHLI, Z.; KNELLER, R. (2016). "Estimating the effects of the container revolution on world trade". Journal of International Economics, 98, pp. 3650.

BREITUNG, J.; PESARAN, M. H. (2007). "Unit roots and cointegration in panels". En MATYAS, L.; SEVESTRE P. (ed.): The Econometrics of Panel Data: Fundamentals and Recent Developments in Theory and Practice. Berlin: Springer.

COTO-MILLÁN, P., INGLADA, CASARES, P., CASTANEDO, M.A.,PESQUERA, SAINZ,R.,FERNÁNDEZ, X.L. (2018). "Demand of port traffic of agricultural food products in Spain". International Journal of Transport Economics. (IN press).

COTO-MILLÁN, P., INGLADA, CASARES, P., CASTANEDO, MATEO, I.; M.A.,PESQUERA, SAINZ,R. (2018). "Demand of port traffic by type of goods in Spain: 1995.I-2007.IV". Maritime Economics and Logistics, 15(4), pp. 444-466.

$\mathrm{CHOI}, \mathrm{I}$, (2001). "Unit root tests for panel data". Journal of International Money and Finance, 20(2), pp. 249-272

FUNG, M. (2002). "Forecasting Hong Kong's container throughput: An error correction model". Journal of Forecasting, 21(1), pp. 69-80.

HUI, E. C.;; SEABROOKE, W.; WONG, G.K. (2004). "Forecasting cargo throughput for the Port of Hong Kong: Error correction model approach". Journal of Urban Planning and Development, 130(4), pp. 195-203.

IM, K. S.; PESARAN, M. H.; SHIN, Y. (2003). "Testing for unit roots in heterogeneous panels". Journal of Econometrics, 115, pp. 53-74.

LUO, M; FAN, L.; LIU, L. (2009). "An econometric analysis for container shipping market". Maritime Policy \& Management, 36(6), pp. 507-523.

PENG, W.Y.; CHU, C.W. (2009). "A comparison of univariate methods for forecasting container throughput volumes". Mathematical and Computer Modelling, 50, pp. 10451057.

PESARAN, M. H.; SHIN, Y.; SMITH, R. (1999). "Pooled men Group estimation of dynamic heterogeneous panels". Journal of the American Statistical Association, 94, pp. 621-634.

RUA, G. (2014). "Diffusion of Containerization". Finance and Economics Discussion Series 2014-88, Board of Governors of the Federal Reserve System (U.S.). 
UNCTAD (2016). Informe sobre el transporte marítimo. Conferencia de las Naciones Unidas sobre Comercio y Desarrollo.

WESTERLUND, J. (2007). "Testing for error correction in panel data". Oxford Bulletin of Economics and Statistics, 69, pp. 709-748. 
